\title{
Influence of Sea Surface Temperature on Outbreak of Ulva prolifera in the Southern Yellow Sea, China
}

\author{
ZHANG Guangzong ${ }^{1}$, WU Mengquan ${ }^{1}$, ZHANG Anding ${ }^{1}$, XING Qianguo ${ }^{2}$, ZHOU Min $^{1}$, ZHAO Deheng $^{1}$, SONG \\ Xiaodong ${ }^{3}$, YU Zhilu ${ }^{4}$ \\ (1. College of Resources and Environmental Engineering, Ludong University, Yantai 264025, China; 2. Yantai Institute of Coastal Zone \\ Research, Chinese Academy of Sciences, Yantai 264003, China; 3. College of Geomatics \& Municipal Engineering, Zhejiang University \\ of Water Resources and Electric Power, Hangzhou 310018, China; 4. Shandong Zhengyuan Digital City Construction Co., Ltd., Yantai \\ 264000, China)
}

\begin{abstract}
In this study, using Moderate Resolution Imaging Spectroradiometer (MODIS) satellite images and environmental satellite CCD images, the spatio-temporal distribution of Ulva prolifera in the southern Yellow Sea during the period of 2011-2018 was extracted and combined with MODIS Level3 Photosynthetically Active Radiation (PAR) product data and Earth System Research Laboratory (ESRL) Sea Surface Temperature (SST) data to analyze their influences on the growth and outbreak of Ulva prolifera. The following conclusions were drawn: 1) comprehensive analysis of Ulva prolifera distribution during the eight-year period revealed that the coverage area of Ulva prolifera typically exhibited a gradually increasing trend. The coverage area of Ulva prolifera reached a maximum of approximately $1714.21 \mathrm{~km}^{2}$ during the eight-year period in late June 2015 . The area affected by Ulva prolifera fluctuated. In mid-July 2014, the area affected by Ulva prolifera reached a maximum of approximately $39020.63 \mathrm{~km}^{2} .2$ ) The average growth rate of Ulva prolifera was positive in May and June but negative in July. During the outbreak of Ulva prolifera, the SST in the southern Yellow Sea tended to increase each month. The SST anomaly and average growth rate of Ulva prolifera were positively correlated in May $\left(R^{2}=\right.$ 0.62), but not significantly correlated in June or July. 3) The variation trends of PAR and SST were approximately the same, and the PAR during this time period maintained a range of $40-50 \mathrm{~mol} /\left(\mathrm{m}^{2} \cdot \mathrm{d}\right)$, providing sufficient illumination for the growth and outbreak of Ulva prolifera. In addition, the abundant nutrients and suitable temperature in the sea area near northern Jiangsu shoal resulted in a high growth rate of Ulva prolifera in May. In summary, the outbreak of Ulva prolifera was closely related to the environmental factors including SST, nutrients, and PAR. Sufficient nutrients and suitable temperatures resulted in a fast growth rate of Ulva prolifera. However, under poor nutrient conditions, even more suitable temperatures were not sufficient to trigger an outbreak of Ulva prolifera.
\end{abstract}

Keywords: Sea Surface Temperature (SST); Photosynthetically Active Radiation (PAR); Ulva prolifera; MODIS (Moderate Resolution Imaging Spectroradiometer); the southern Yellow Sea

Citation: ZHANG Guangzong, WU Mengquan, ZHANG Anding, XING Qianguo, ZHOU Min, ZHAO Deheng, SONG Xiaodong, YU Zhilu, 2020. Influence of Sea Surface Temperature on Outbreak of Ulva prolifera in the southern Yellow Sea, China. Chinese Geographical Science, 30(4): 631-642. https://doi.org/10.1007/s11769-020-1129-9

Received date: 2019-06-26; accepted date: 2019-10-21

Foundation item: Under the auspices of Natural Science Foundation of Shandong (No. ZR2019MD041), National Natural Science Foundation of China (No. 41676171), Qingdao National Laboratory for Marine Science and Technology of China (No. 2016ASKJ02), Natural Science Foundation of Shandong (No. ZR2015DM015), Development and Construction Funds Project of National Independent Innovation Demonstration Zone in Shandong Peninsula (No. ZCQ17117)

Corresponding author: WU Mengquan. E-mail: ld_wmq@1du.edu.cn; ZHANG Anding. E-mail: andingzhang@sina.com

(C) Science Press, Northeast Institute of Geography and Agroecology, CAS and Springer-Verlag GmbH Germany, part of Springer Nature 2020 


\section{Introduction}

Green tide is ecological anomalies consisting of marine macroalgae outbreaks and aggregation that endanger the balance of marine ecosystems (Wu, 2015). In recent years, dominated by Ulva prolifera, severe green tide disasters have occurred in the southern Yellow Sea of China, which have had a considerable impact on the tourism, aquaculture and ecological environment of coastal cities in China (Mao et al., 2018). Every year, China has to invest many human, material and financial resources in the monitoring and control Ulva prolifera. Due to the substantial impact of green tide disasters on the coastal ecological environment and economic development, Ulva prolifera has become a popular research topic worldwide (Liu et al., 2009; 2016; $\mathrm{Xu}$ et al., 2014). Given its large-scale, rapid and real-time dynamic observation capabilities (Mao et al., 2020), remote sensing technology plays an important role in monitoring outbreaks of Ulva prolifera and studying their influence on the marine environment.

To date, numerous studies of Ulva prolifera have been performed. In terms of the origin of Ulva prolifera, Keesing et al. (2011) and Song et al. (2015) found that the occurrence of green tides in the Yellow Sea was closely related to the expansion of seaweed cultivation in the sea area near the shoal of northern Jiangsu Province, because the culture rafts of Porphyra provide ideal conditions for the germination of Ulva prolifera's microcosmic propagules. Ding et al. (2009) and Li et al. (2009) comparatively analyzed the meteorological and hydrological conditions in the sea areas of Jiangsu Province and Qingdao during outbreaks of Ulva prolifera and found that they were closely related to factors such as eutrophication, illumination, temperature and precipitation in nearshore waters. To analyze and predict the drifting path of Ulva prolifera, Lee et al. (2011), Qiao et al. (2011) and Zhao et al. (2018) simulated the drifting path of Ulva prolifera with a coupled three-dimensional surface wave-tide-circulation model and Lagrangian particle tracing experiments, which revealed that sea surface wind and sea surface currents jointly affected the drifting of Ulva prolifera, and the simulated path was similar to the actual drifiting of Ulva prolifera. In studies on the competitive relationship between Ulva prolifera and microalgae, Xing et al. (2015) and Sun et al. (2018) inverted the chlorophyll-a concentration in the
Yellow Sea based on MODIS data and Geostationary Ocean Color Imager (GOCI) data respectively, and then analyzed the relationship between chlorophyll-a and Ulva prolifera. Their results indicate that outbreaks of Ulva prolifera were negatively correlated with planktonic microalgae in the same sea area. In a study on the use of remote sensing to monitor and extract information on Ulva prolifera, Wang et al. (2014) used MODIS data to evaluate the accuracy of five different vegetation indexes in the extraction of Ulva prolifera information and found that an algorithm based on the Normalized Difference Vegetation Index (NDVI) was the most accurate and stable. Qi et al. (2016) statistically analyzed the outbreak area of Ulva prolifera over multiple years in the Yellow Sea using MODIS data, determining that 2015 was the year with the largest outbreak area of Ulva prolifera in recent years.

The growth of Ulva prolifera, a macroalgae, is subject to the influence of illumination and temperature. Studies based on Ulva prolifera cultured in the laboratory reveal that, under suitable illumination conditions, Ulva prolifera has a good population growth rate in a temperature range of $5^{\circ} \mathrm{C}$ to $30^{\circ} \mathrm{C}$, with a suitable temperature range of $18^{\circ} \mathrm{C}$ to $23^{\circ} \mathrm{C}$, and it is adaptable to a relatively wide range of temperatures (Taylor et al., 2001; Fan et al., 2015; Wang et al., 2018). Most previous studies focused on the relationship between the early growth stage of Ulva prolifera and Sea Surface Temperature (SST), but few focused on the relationship between the whole life cycle of Ulva prolifera and SST. In particular, in studies on the relationship between the monthly average growth rate of Ulva prolifera and monthly average SST, the effect of Photosynthetically Active Radiation (PAR) on the two factors has not been considered. Therefore, in this study, we combine MODIS satellite data and high-resolution environmental satellite (HJ-1A/B) CCD data via the algorithm based on NDVI to extract temporal and spatial distribution information for Ulva prolifera from 2011 to 2018. The aims of this study are to: 1) explore the temporal and spatial characteristics of SST in the southern Yellow Sea; 2) analyze the influence of SST on the growth rate of Ulva prolifera during 2011-2018 in the study area; and 3) explore the relationship between SST and PAR in the natural growth environment. We hope to provide a scientific basis for monitoring Ulva prolifera. 


\section{Data and Methods}

\subsection{Study area}

The southern Yellow Sea $\left(119^{\circ} \mathrm{E}-123^{\circ} \mathrm{E}, 32^{\circ} \mathrm{N}-37^{\circ} \mathrm{N}\right)$ was selected as the study area (Fig. 1). This is a semi-enclosed shallow sea area of approximately 309 $000 \mathrm{~km}^{2}$ located near Shandong Peninsula and Jiangsu shoal, and the turbidity of water in the study area is highly variable (Sun et al., 2017). In particular, Jiangsu shoal has been developed into the largest Porphyra cultivation base in China because of its unique geographic conditions and local microclimate. The complex hydrological conditions of the southern Yellow Sea, including the influence of the warm current in the Yellow Sea, the tidal current along the Yellow Sea and fresh water from the Yangtze River, result in a large degree of variability in the temporal and spatial distributions of nutrients.

\subsection{Data sources}

The remote sensing data used in this study primarily included MODIS image data, environmental satellite image data (HJ-1A/B CCD), MODIS Level3 PAR data and SST data from the National Oceanic and Atmospheric Administration (NOAA) Earth System Research Laboratory (ESRL). The PAR data are tertiary product data provided by the SeaWiFS website (https://oceancolor.gsfc.nasa. gov), and the ESRL SST data are from the ESRL (http://www.esrl.noaa.gov/psd). The MODIS satellite data cover a wide width and have high temporal resolution (http://ladsweb.modaps.eosdis. nasa.gov), two scenes are acquired every day, and the first and second bands have a spatial resolution of $250 \mathrm{~m}$. The environmental satellite (http://www.cresda.com/CN) data include four bands in a range from visible to near-infrared light, with a spatial resolution of $30 \mathrm{~m}$, enabling more accurate acquisition of information about Ulva prolifera. Because remote sensing satellites are affected by factors such as time period and weather, it is very difficult to sufficiently satisfy the time and quantity requirements for the extraction of Ulva prolifera information from single-source satellite data. Therefore, in this study, we comprehensively used MODIS and environmental satellite remote sensing image data. By extracting the coverage area of Ulva prolifera from the two types of satellite images on the same date, we established a linear relationship of $y=2.266 x+$ $1.730\left(R^{2}=0.991\right)$. This equation was used to calculate the coverage area of Ulva prolifera from the MODIS data to complete the missing environmental satellite data, thus enabling better extraction of Ulva prolifera distribution information over multiple years (Zhang et al., 2018). The acquisition dates of the multisource remote sensing satellite images during 2011-2018 are reported in Table 1.

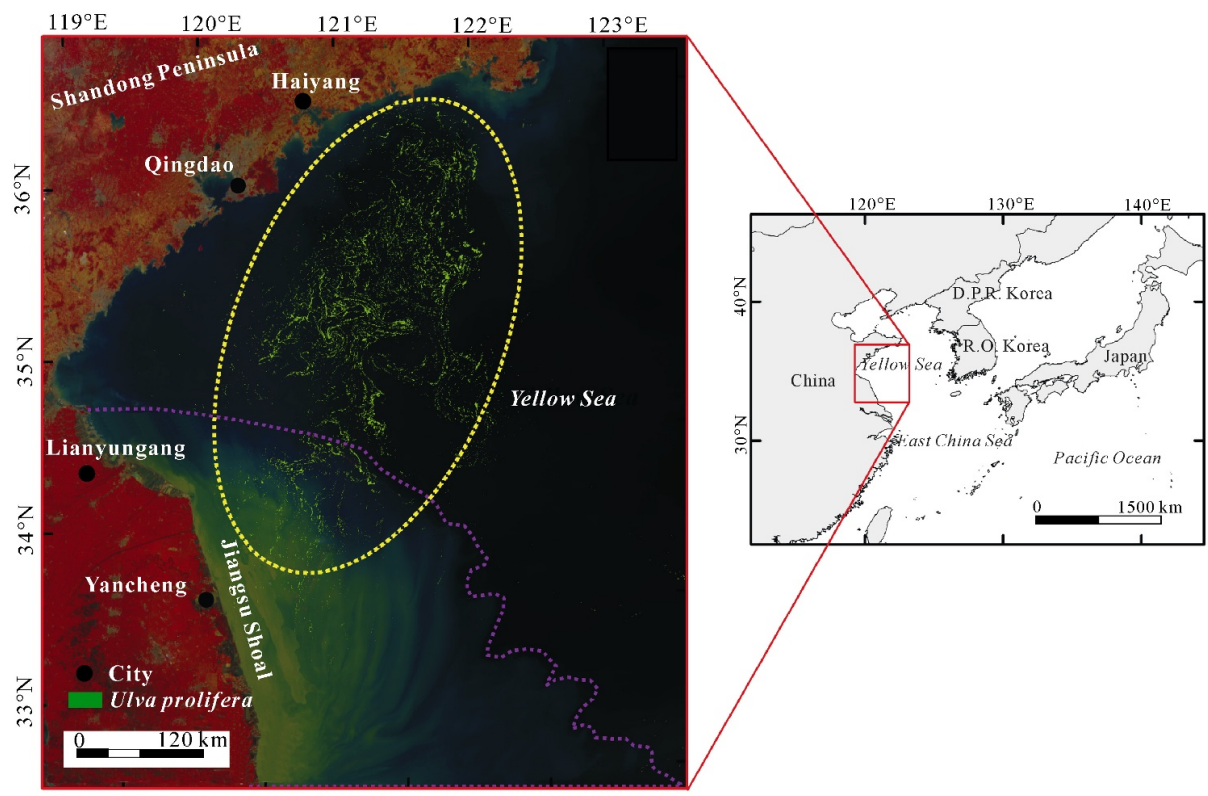

Fig. 1 Location of the study area and the exemplified spatial distribution of Ulva prolifera (green slicks within the yellow dashed circle) extracted from HJ (Huanjing)-1A/B images on June 21, 2015. The purple dashed line is the dividing line between the southeastern part of Shandong Peninsula and the waters of the Jiangsu Shoal 
Table 1 The date of satellite images of Ulva prolifera from 2011 to 2018 in the southern Yellow Sea, China

\begin{tabular}{lll}
\hline Year & HJ-1A/B CCD image date & MODIS image date \\
\hline 2011 & $05-29,07-26,08-09$ & $06-13,06-20,07-04$ \\
2012 & $05-17,06-12,06-22,08-06$ & $05-26,07-11$ \\
2013 & $05-22,06-26,07-24$ & $05-13,06-03,06-29,08-05$ \\
2014 & $05-22,07-09,08-01$ & $05-12,05-26,06-06,06-12,6-30$, \\
2015 & $05-10,07-14$ & $07-14,07-28$ \\
2016 & $05-17,07-14,07-30$ & $05-20,06-21,07-04,07-30$ \\
2017 & $05-20,07-05$ & $05-25,06-01,06-13,06-25,07-02$ \\
2018 & $06-24$ & $06-17,06-27$ \\
\hline
\end{tabular}

Note: MODIS represents Moderate Resolution Imaging Spectroradiometer

\subsection{Research method and data processing}

Numerous studies have found that the NDVI can also extract Ulva prolifera information in the presence of thin clouds and has the greatest Ulva prolifera detection capability at different growth stages (Wang et al., 2014). Therefore, the NDVI algorithm was adopted in this study to extract Ulva prolifera information. The calculation formula of the NDVI algorithm is as follows:

$N D V I=\left(R_{\mathrm{NIR}}-R_{\mathrm{RED}}\right) /\left(R_{\mathrm{NIR}}+R_{\mathrm{RED}}\right)$

where $R_{\text {NIR }}$ and $R_{\text {RED }}$ denote the remote sensing reflectivity in the near-infrared band and infrared band, respectively. The NDVI values of water and vegetation are negative and positive, respectively. We used the NDVI threshold to partition and differentiate the distribution regions of Ulva prolifera and then extracted the area of Ulva prolifera by setting a reasonable threshold T. When extracting information from remote sensing images by the NDVI algorithm, the threshold for vegetation is usually 0 , but owing to the influences of mixed pixels, aggregation density, and suspension status of Ulva prolifera at different stages, the threshold value may not be 0 (Cai et al., 2014). We empirically determined the best NDVI threshold for extracting Ulva prolifera by extensive experiments and visual interpretation. We checked all the HJ-1 CCD and MODIS images available for the study area and found that the NDVI threshold of Ulva prolifera was within $0-0.15$ for MODIS (the lowest threshold value was -0.15 on July 30 ) and $0-0.4$ for HJ-1 (Sun et al., 2018). The specific process of extracting Ulva prolifera information is presented in a flowchart (Fig. 2).

The extracted distribution information for Ulva prolifera was vectorized with ENVI remote sensing processing software and then imported into ArcGIS. We conducted a cluster analysis of the coverage area data for Ulva prolifera. The scattered patches of Ulva prolifera were aggregated into concentrated, large patches, and the aggregated patches represented the influence area of Ulva prolifera. The tertiary monthly MODIS satellite PAR product data and monthly SST data obtained in this study had a spatial resolution of $4 \mathrm{~km}$. By

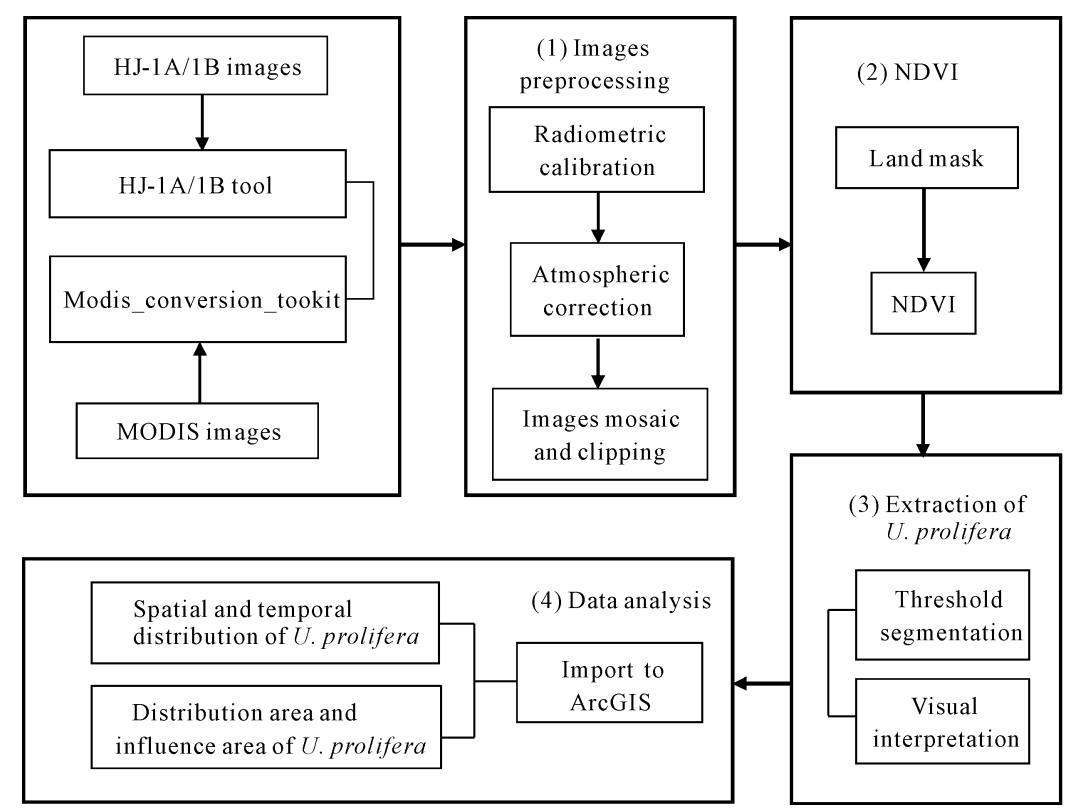

Fig. 2 Flow chart of data processing 
using ocean color remote sensing processing software (SeaDAS), we subjected the data to regional clipping, segmentation and land masking. The generated images were imported into ArcGIS and combined with variation in spatial location during the growth and outbreak of Ulva prolifera for statistical analysis and processing.

\section{Results}

\subsection{Temporal and spatial distributions of Ulva prolifera}

We used ArcGIS software to process the Ulva prolifera information and obtain the temporal and spatial distributions of Ulva prolifera in the southern Yellow Sea during 2011-2018 (Fig. 3). Due to limited data, we selected four representative distribution maps of different Ulva prolifera growth stages every year to reflect the temporal and spatial distributions and variation in Ulva prolifera in the southern Yellow Sea, China. With the obtained quantitative relation, we calculated the coverage area of Ulva prolifera, which was extracted from the MODIS data, to replace the missing HJ satellite data. Moreover, by combining remote sensing satellite monitoring data for Ulva prolifera over multiple years, we obtained monitoring information for Ulva prolifera during 2011-2018, as presented in Table 2. The coverage area of Ulva prolifera represents the real distribution area, and the area of influence represents aggregation scattered fragments of the Ulva prolifera coverage area into large fragmented areas.

By analyzing the temporal and spatial distributions of Ulva prolifera in the southern Yellow Sea during the period of 2011-2018, we found that Ulva prolifera blooms exhibited five stages, namely emergence, development, outbreak, decline, and disappearance, during the eight-year period (Wu et al., 2014). Moreover, using the remote sensing approach, we discovered for the first time that the region of Ulva prolifera was located in the sea area near Jiangsu shoal. The interannual variation in Ulva prolifera drifting direction was approximately uniform. Specifically, Ulva prolifera found early in the year in the sea area near Jiangsu shoal moves northward to the sea area near Shandong Peninsula, driven by surface sea currents and sea surface wind. In June, Ulva prolifera undergoes large-scale growth resulting in an outbreak, aggregating in a wide swath of sea area along the coast of Rizhao and Qingdao City and leading to a green tide disaster. From late June to early July, Ulva prolifera begins to enter a declining stage, whereby the area affected by Ulva prolifera gradually decreases before the green tide eventually dies out in the sea area near Yantai and Weihai cities. The largest area of Ulva prolifera during the eight-year period was observed in $2015\left(1752.76 \mathrm{~km}^{2}\right)$; at this time, the range of the area of influence was at a maximum, extending to the sea area near Weihai, Shandong Province, consistent with the research results of Qi et al. (2016). In addition, the area in 2012 was the smallest during the eight-year period during the Ulva prolifera outbreak. We used remote sensing images to monitor the disappearance of Ulva prolifera on August 6, 2012, when the influence range of Ulva prolifera reached its farthest extent; the sea area near Yantai City, Shandong Province.

By analyzing the monitoring dates of Ulva prolifera during the period of 2011-2018, we found that the coverage area of Ulva prolifera reached its eight-year maximum extent of approximately $1752.76 \mathrm{~km}^{2}$ on June 21, 2015. Beginning in 2016, the coverage area of Ulva prolifera gradually decreased each year. In a given year, the coverage area of Ulva prolifera reached a peak in

Table 2 Remote sensing monitoring information for Ulva prolifera from 2011 to 2018

\begin{tabular}{cccccc}
\hline Year & Initial discovery time & Last discovery time & Time of duration $(\mathrm{d})$ & Maximum distribution area $\left(\mathrm{km}^{2}\right)$ & Maximum influence area $\left(\mathrm{km}^{2}\right)$ \\
\hline 2011 & $05-29$ & $08-09$ & 73 & 392.46 & 8002.50 \\
2012 & $05-17$ & $08-06$ & 82 & 221.68 & 20076.51 \\
2013 & $05-13$ & $08-05$ & 85 & 690.74 & 14945.82 \\
2014 & $05-12$ & $08-01$ & 82 & 621.29 & 39020.63 \\
2015 & $05-10$ & $08-05$ & 88 & 1752.76 & 34784.11 \\
2016 & $05-17$ & $07-30$ & 75 & 1487.81 & 31979.11 \\
2017 & $05-20$ & $07-05$ & 47 & 910.44 & 15920.08 \\
2018 & $06-07$ & $07-15$ & 39 & 1151.81 & 12145.53 \\
\hline
\end{tabular}



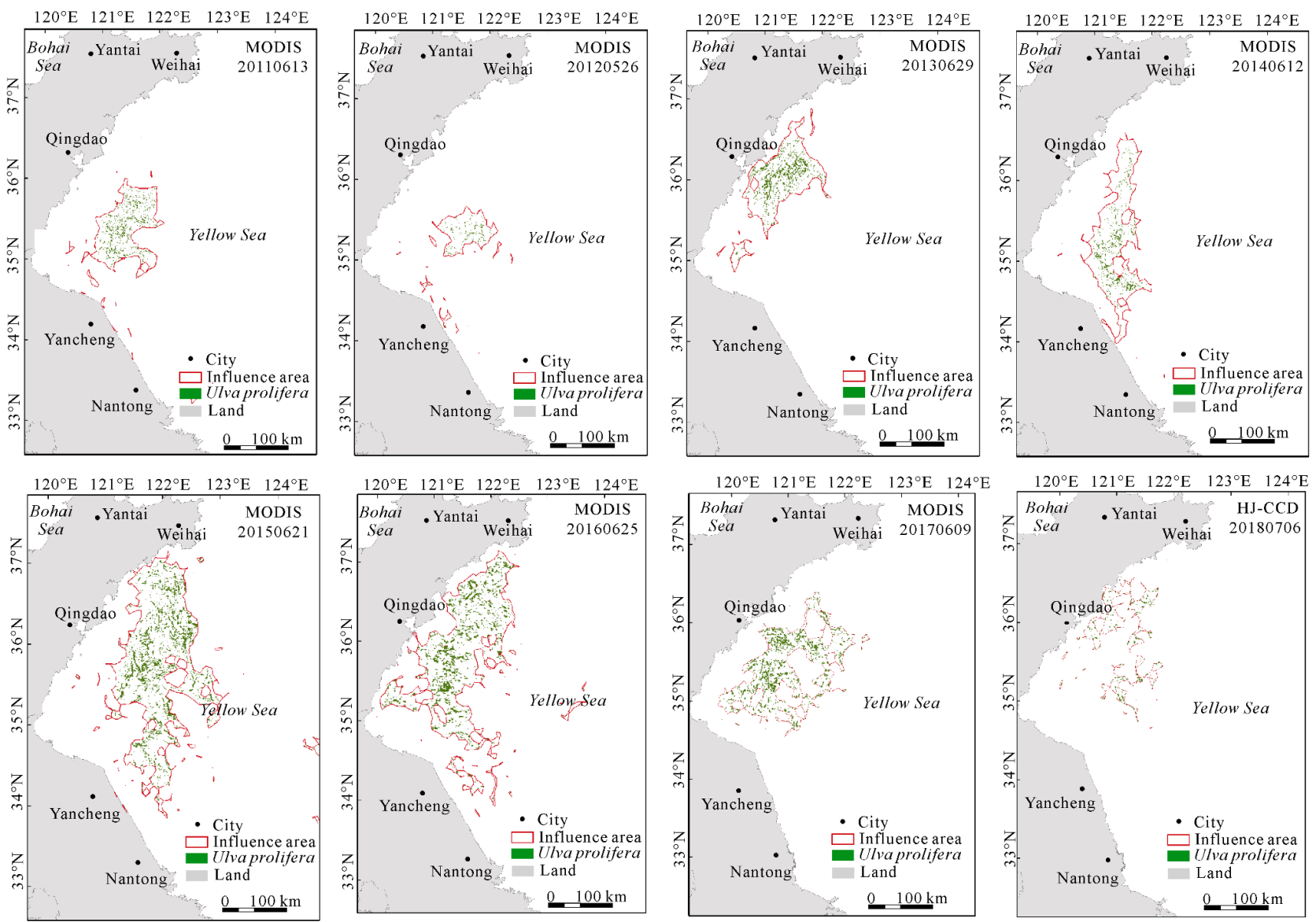

$120^{\circ} \mathrm{E} \quad 121^{\circ} \mathrm{E} \quad 122^{\circ} \mathrm{E} \quad 123^{\circ} \mathrm{E} \quad 124^{\circ} \mathrm{E}$
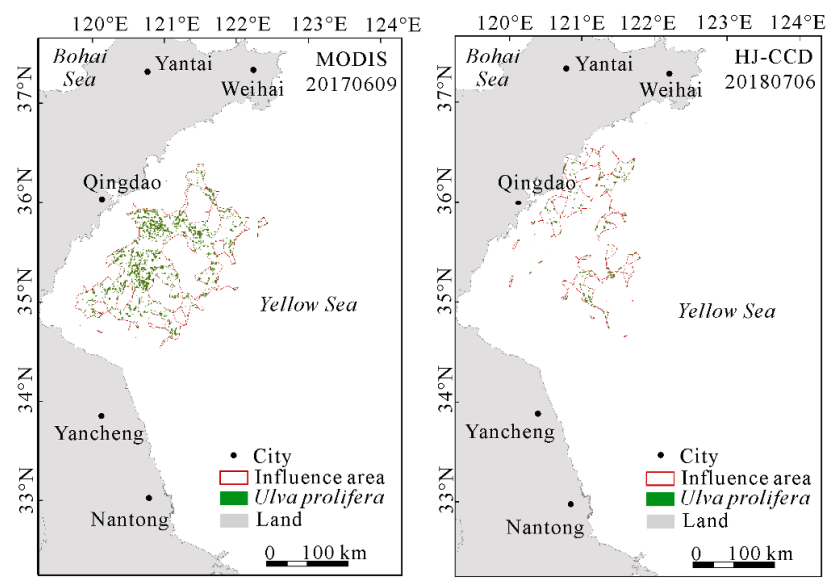

Fig. 3 The spatial and temporal distribution of Ulva prolifera extracted from Moderate Resolution Imaging Spectroradiometer (MODIS) and HJ-1A/B images in the southern Yellow Sea during 2011-2018

June. From May to June, the coverage area of Ulva prolifera tended to gradually increase. The earliest detection date of Ulva prolifera was later in 2018 than in the previous seven years on the basis of remote sensing images, and the large-scale area of Ulva prolifera that aggregated and floated in the sea area near Jiangsu shoal was significantly reduced in 2018 compared with 2017 . This change was related to the early deployment of measures to prevent and control Ulva prolifera green tide in Jiangsu Province in 2018. Surveillance, monitoring, salvage, and disposal of Ulva prolifera during green tide was thoroughly organized and performed. Removal of Ulva prolifera epiphyte on Porphyra culture rafts and Ulva prolifera floating in aquiculture area was made a priority. During the eight-year study period, the area affected by Ulva prolifera exhibited fluctuations. On July 14, 2014, the total area affected by Ulva prolifera reached the eight-year maximum of approximately $39020.63 \mathrm{~km}^{2}$. Due to the influence of different levels of dispersion for the Ulva prolifera patches, the variation in the area affected by Ulva prolifera was not completely consistent with that in the coverage area of this species. When the area affected by Ulva prolifera reached its maximum size, the coverage area of Ulva prolifera did not simultaneously reach a maximum.

By calculating the coverage area of Ulva prolifera in the period of 2011-2018 (http://ncs.mnr.gov.cn/), we obtained the average growth rate of Ulva prolifera from May to July during the eight-year study period (Table 3). The growth rate was calculated by the daily rate of change in the Ulva prolifera coverage area. The initial month in which Ulva prolifera appeared during the period of 2011-2017 was May, whereas in 2018 it was June. Therefore, in this study, no growth rate was obtained for Ulva prolifera in May 2018. Because remote sensing satellite images are affected by factors such as weather, Ulva prolifera monitoring was not continuous over some of the dates, resulting in certain errors in the 
Table 3 The average growth rate of Ulva prolifera during May-July from 2011 to 2018

\begin{tabular}{cccc}
\hline Year & $\begin{array}{c}\text { Average growth rate } \\
\text { in May }\left(\mathrm{km}^{2} / \mathrm{d}\right)\end{array}$ & $\begin{array}{c}\text { Average growth rate } \\
\text { in June }\left(\mathrm{km}^{2} / \mathrm{d}\right)\end{array}$ & $\begin{array}{c}\text { Average growth rate } \\
\text { in July }\left(\mathrm{km}^{2} / \mathrm{d}\right)\end{array}$ \\
\hline 2011 & 21.77 & -11.20 & -12.25 \\
2012 & 13.36 & 1.89 & -2.03 \\
2013 & 3.34 & 12.88 & -16.96 \\
2014 & 26.01 & 4.42 & -10.41 \\
2015 & 23.73 & -1.65 & -14.34 \\
2016 & 26.29 & 7.63 & -11.19 \\
2017 & 60.62 & 0.45 & -11.07 \\
2018 & - & 9.19 & -3.71 \\
Average & 25.02 & 2.95 & -10.25 \\
\hline
\end{tabular}

average growth rate of Ulva prolifera. The analysis showed that the average growth rate of Ulva prolifera in May during the eight-year period was positive. In this month, Ulva prolifera was in a large-scale growth stage. In June, the average growth rate of Ulva prolifera declined from that in May. Specifically, the average growth rate of Ulva prolifera was negative in both 2011 and 2015, but it was still at the outbreak phase throughout the month. In July, the average growth rate of Ulva prolifera was negative, and Ulva prolifera severely declined and died out in this month during 2011-2018.

\subsection{Temporal and spatial distributions of SST}

In this study, ArcGIS software was used to conduct spatial interpolation of ESRL SST data from 2011-2018 in the southern Yellow Sea. Then, we obtained monthly average SST maps for May-August of each year (Fig. 4).

By analyzing the temporal and spatial distributions of average SST in the southern Yellow Sea during May to August in 2011-2018, we found that the average SST in May during the eight-year study period was approximately $12^{\circ} \mathrm{C}-18^{\circ} \mathrm{C}$; specifically, the SST in 2011 was approximately $15^{\circ} \mathrm{C}$, which was the lowest value in the eight-year period. In contrast, the SST in 2017 was approximately $17^{\circ} \mathrm{C}$, which was the highest value in the eight-year period. There was an obvious difference in SST in May between the coasts of Shandong Peninsula and the Jiangsu shoal; specifically, the SST in the sea area near Jiangsu shoal was approximately $14^{\circ} \mathrm{C}-17^{\circ} \mathrm{C}$ in May, which was higher than the average SST of $14^{\circ} \mathrm{C}-16^{\circ} \mathrm{C}$ in the sea area along the coast of Shandong Peninsula in May. In June, the SST in the southern Yellow Sea area typically increased, and the average SST in June for the eight-year study period was approximately $18^{\circ} \mathrm{C}-21^{\circ} \mathrm{C}$. SST in 2011 was approximately $18.5^{\circ} \mathrm{C}$, which was the lowest during the eight-year period, and SST was approximately $21^{\circ} \mathrm{C}$ in 2017 , which was the highest during the eight-year period. The SST in the sea area near Shandong Peninsula and Jiangsu shoal ranged from $19^{\circ} \mathrm{C}-22^{\circ} \mathrm{C}$. The difference in SST between the two locations in June was significant in May but not in June. In July, the SST in the Yellow Sea area increased again to $24^{\circ} \mathrm{C}-26^{\circ} \mathrm{C}$; a comparison of interannual SST in the study area during the same month showed that the SST in July 2015 , measuring $23^{\circ} \mathrm{C}$, was the lowest during the eight-year period. The SST in July 2017 was $27^{\circ} \mathrm{C}$, which was the highest during the eight-year period. With remote sensing images, Ulva prolifera could not be detected in early July 2017, which was the earliest disappearance date of Ulva prolifera in the recent eight-year period. In August, the SST in the southern Yellow Sea was approximately $25^{\circ} \mathrm{C}-29^{\circ} \mathrm{C}$, and the SST in the sea area near Shandong Peninsula and Jiangsu shoal increased again compared to that in July. The interannual variation in SST was not significant in the study area.

The trend of interannual variation in SST in the southern Yellow Sea was consistent during the eight-year period, and the temperature increased each month from May to August. The difference in interannual variation in SST was not significant. However, under the influence of global warming, the interannual SST in the southern Yellow Sea area has exhibited a gradually increase in recent years (Park et al., 2015; Liu et al., 2018).

\section{Discussion}

\subsection{Influence mechanism of SST on Ulva prolifera}

To further study and analyze the mechanism underlying the influence of SST on Ulva prolifera, we conducted SST anomaly detection with the SST data collected in the study area (Jin et al., 2018). With correlation analysis, we quantified the relationship between SST from May to July in 2011-2018 and the average growth rate of Ulva prolifera during the same time period in the southern Yellow Sea, China (Figs. 5, 6). Because the growth rate of Ulva prolifera differs among growth phases and varied among years during the $8 \mathrm{yr}$, we analyzed the influence of SST on the growth rate of Ulva prolifera by month. 

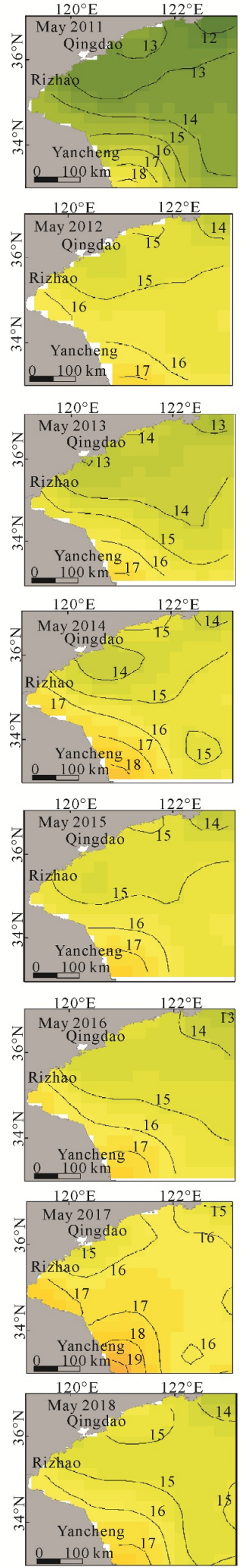
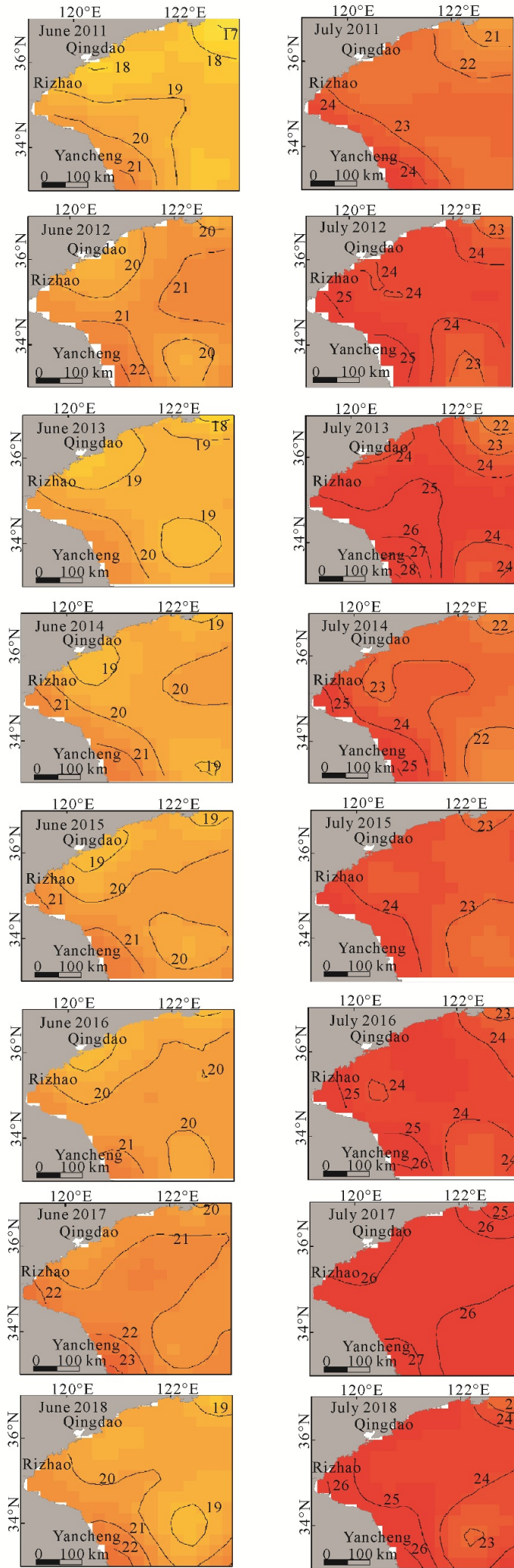
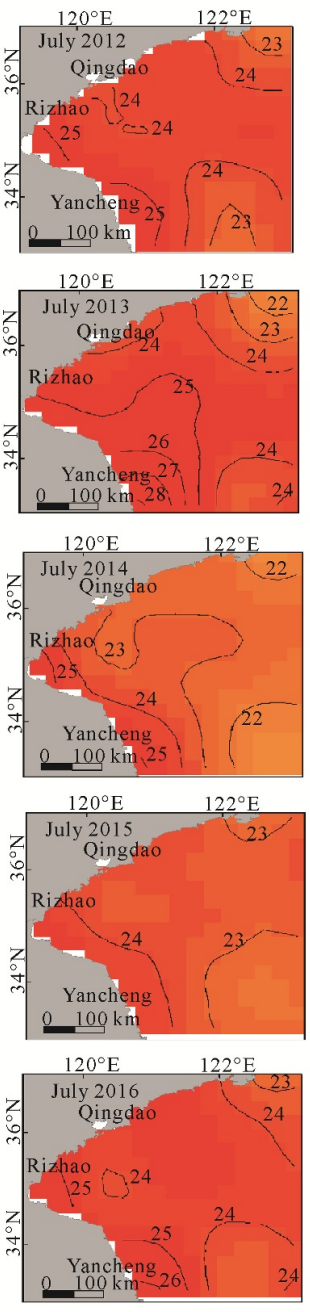$$
120^{\circ} \mathrm{E}
$$

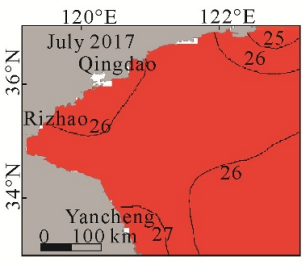

$120^{\circ} \mathrm{E}$

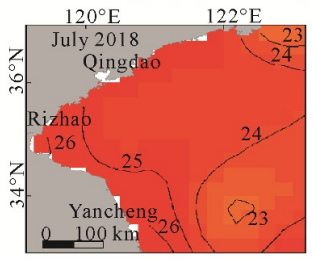

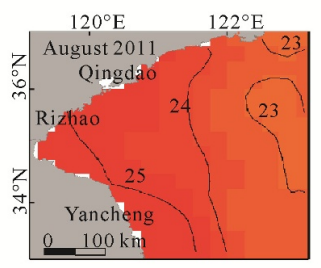
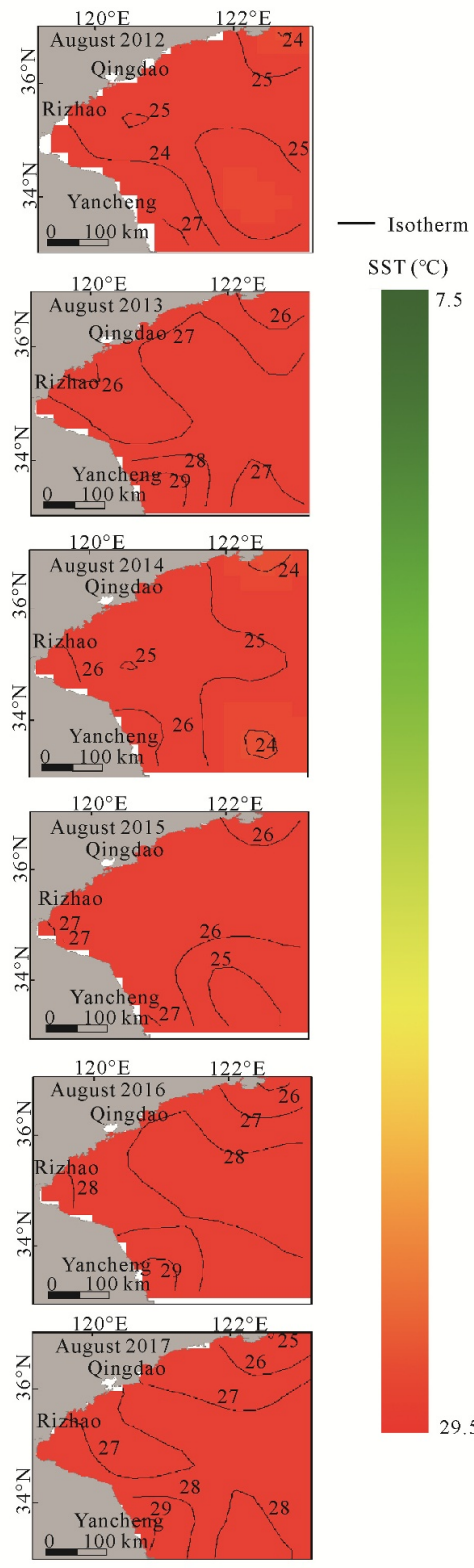

$120^{\circ} \mathrm{E}$

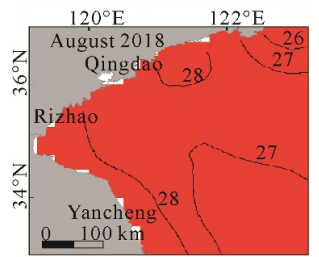

Fig. 4 The average sea surface temperature (SST) in the southern Yellow Sea during May-August from 2011 to 2018 


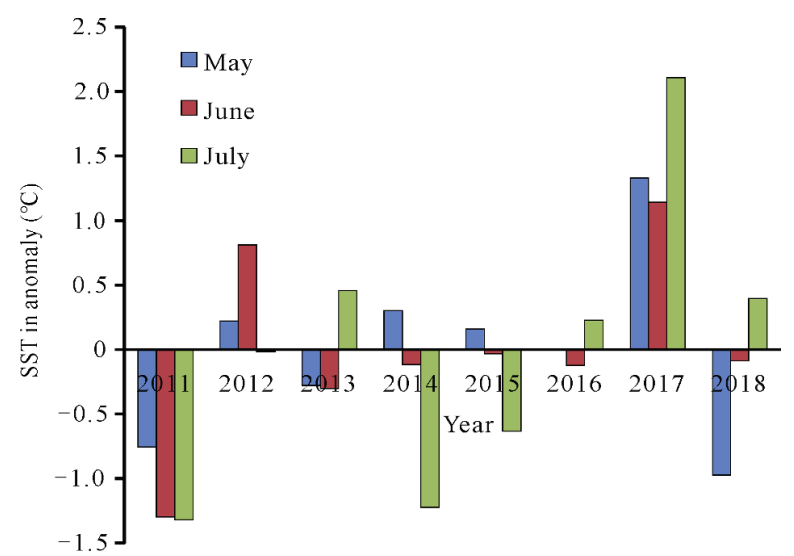

Fig. 5 Monthly anomalies of sea surface temperature (SST) during May-July from 2011 to 2018

Figs. 6a-6c show the association between the SST anomaly and the average growth rate of Ulva prolifera in May, June and July (specifically, Fig. 6a is for 2011-2017), respectively. The SST anomaly was significantly and positively correlated with the average growth rate of Ulva prolifera in May in each of the eight years $\left(R^{2}=0.62, P<0.05\right)$. The average SST in the study area was approximately $15.73^{\circ} \mathrm{C}$ in May during the eight-year period. In May, the average growth rate of Ulva prolifera increased with an increase in SST anomaly. In 2017 , when the SST was approximately $17.06^{\circ} \mathrm{C}$, the average growth rate of Ulva prolifera reached a maximum in May, which was consistent with the result of Fan (Fan et al., 2012). Based on field survey data, those authors found the water temperature during the formation of large-scale Ulva prolifera outbreak was approximately $17^{\circ} \mathrm{C}$. In June and July, the correlation between the SST anomaly and the average growth rate of Ulva prolifera was relatively weak. In the two months, there was no strong association between the change in SST and the average growth rate of Ulva pro-
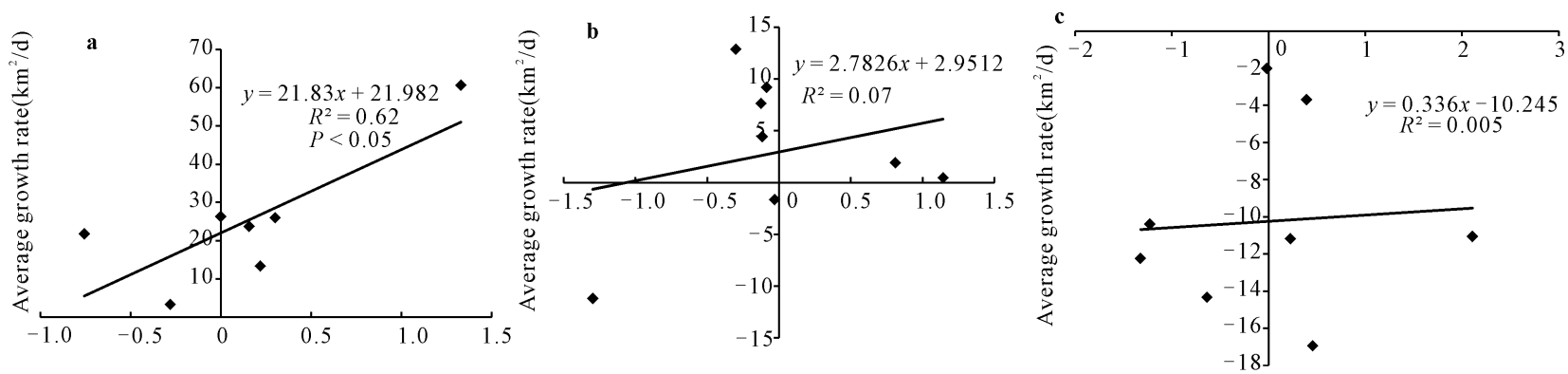

Fig. 6 The correlation between monthly sea surface temperature (SST) anomaly and average growth rate of Ulva prolifera (a, b, c represents May, June and July, respectively) lifera in the study area.

In contrast to Ulva prolifera cultured under laboratory conditions (Zhang et al., 2012; Mao et al., 2019), Ulva prolifera in the sea area near Jiangsu shoal exhibited a relatively high growth rate despite most temperatures being lower than the suitable temperature range. This phenomenon can likely be attributed to the abundant nutrients and suitable meteorological conditions in the sea area near Jiangsu shoal (Cui et al., 2014; Wei et al., 2016). Moreover, the microscopic propagules of Ulva prolifera have a high germination rate under temperatures of $10^{\circ} \mathrm{C}-25^{\circ} \mathrm{C}$. These conditions lead to large-scale outbreak of Ulva prolifera in May, indicating that May is the key month for outbreaks of this species (Jin et al., 2018). In June and July, Ulva prolifera drifted in the northeast direction and entered the sea area near Shandong Peninsula, driven by the surface current and sea surface wind (Yi et al., 2010). During this process, the SST in the study area gradually increased and reached a range of temperatures suitable for Ulva prolifera. However, based on the analysis in Fig. 6, the change in SST did not cause a high growth rate of Ulva prolifera, likely because of the consumption of nutrients by the large propagation of Ulva prolifera in this region (Shi et al., 2015).

\subsection{Relationship between PAR and SST}

Irradiance and temperature are two major factors influencing algal growth, and they also affect the release of spores and germination (Zhang et al., 2016). Moreover, temperature is directly related to irradiance (Wild et al., 2005). During periods of growth and outbreak, Ulva prolifera converts carbon dioxide into organic matter through photosynthesis, providing the basis for its growth and material accumulation (Collini et al., 2010). The portion of solar radiation used by vegetation for 
photosynthesis is known as PAR (Zhu et al., 2010). As the main energy source, solar radiation continuously affects the growth of Ulva prolifera. When the illumination intensity is within a certain range, photosynthesis increases with illumination intensity. However, when the illumination intensity exceeds a certain range, photoinhibition occurs. The rate of algal photosynthesis also varies among temperatures (Fu et al., 2018). Therefore, to investigate the relationship between PAR and SST in the southern Yellow Sea, we conducted a simple comparative analysis of PAR and SST in this area (Fig. 7).

Figs. 7a-7c show that the variation trends of PAR and SST were approximately the same in the study area during May-July over the eight-year period. With an increase or a decrease of PAR, SST also increased or decreased correspondingly, which was consistent with the research results of Wang (Wang et al., 2010). Cui et al. (2015) found that the fluctuation of light intensity in the Yellow Sea was more than $300 \mu \mathrm{mol} /\left(\mathrm{m}^{2} \cdot \mathrm{s}\right)$ on sunny days and the maximum light intensity was approximately $2000 \mu \mathrm{mol} /\left(\mathrm{m}^{2} \cdot \mathrm{s}\right)$. Under the influence of global warming, light intensity trends to increase each year. In the southern Yellow Sea, PAR maintained a range of $40-50 \mathrm{~mol} /\left(\mathrm{m}^{2} \cdot \mathrm{d}\right)$ during May-July. Thus, PAR in the study area during the eight-year period remained somewhat stable, and suitable illumination provided energy for the growth and outbreaks of Ulva prolifera. The amount of solar radiation absorbed by Ulva prolifera during photosynthesis was stable, but the correlation between SST and the average growth rate of Ulva prolifera varied. The most significant correlation between the two factors occurred in May because there was more suspended sediment and lower seawater transparency near the Jiangsu shoal than observed during other months. This attenuated light intensity and incident light penetration improved algal metabolism and productivity.
In addition, the sea area near the northern Jiangsu shoal was characterized by abundant nutrients, which caused SST to become the key environmental factor limiting the growth of Ulva prolifera in May (Jin et al., 2018). In June and July, free-floating green algae are exposed to extremely high irradiance levels when they continuously float on the surface, which causes photoinhibition and damage by ultraviolet radiation (Kim et al., 2011). Meanwhile, the photosynthetic rate of Ulva prolifera decreases. Although SST is within the optimum temperature range of Ulva prolifera at this time, the growth of this species is restricted by deficient nutrient conditions and different environmental factors compared with those occurring in the Jiangsu shoal, such that the correlation between SST and the average growth rate of Ulva prolifera is not significant.

\section{Conclusions}

In this study, by using MODIS and HJ-1A/B CCD images, we extracted spatiotemporal distribution information for Ulva prolifera and its monthly average growth rate in the South Yellow Sea during 2011-2018. Based on analysis the influence of SST on Ulva prolifera, PAR was examined to explore the relationship between the three factors. The following conclusions can be drawn:

(1) Comprehensive analysis of the Ulva prolifera distribution during the eight-year period revealed that the coverage area of Ulva prolifera tended to gradually expand. The coverage area of Ulva prolifera reached a maximum of approximately $1714.21 \mathrm{~km}^{2}$ during the eight-year period in late June 2015 . The area affected by Ulva prolifera fluctuated. In mid-July 2014, the area affected by Ulva prolifera reached the eight-year maximum of approximately $39020.63 \mathrm{~km}^{2}$.
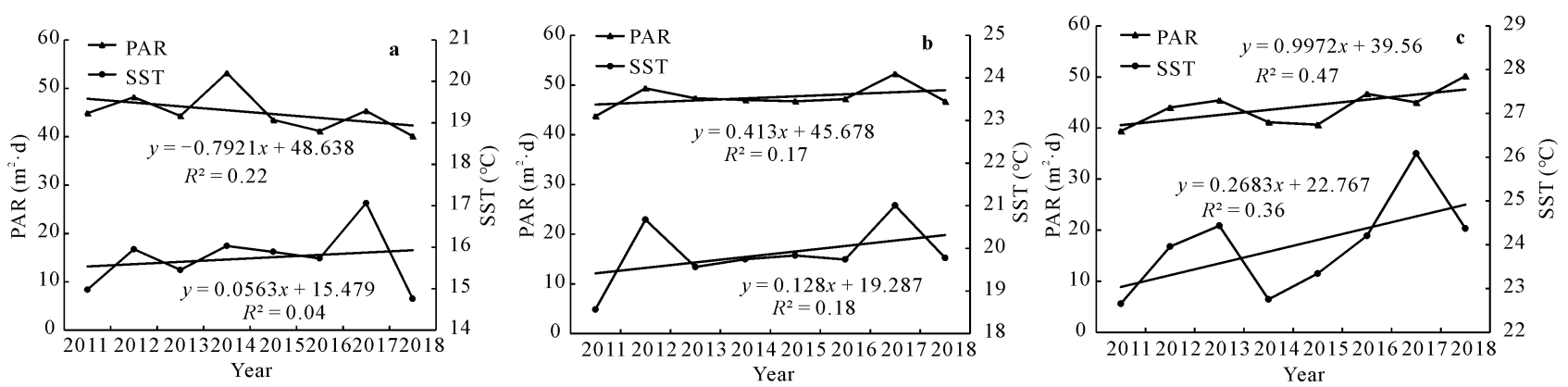

Fig. 7 Comparison photosynthetically active radiation (PAR) and sea surface temperature in the southern Yellow Sea from May to July (a-c) during 2011-2018 
(2) The average growth rate of Ulva prolifera was positive in May and June but negative in July. During the outbreak of Ulva prolifera, the SST in the southern Yellow Sea tended to increase each month. The SST anomaly and average growth rate of Ulva prolifera were positively correlated in May $\left(R^{2}=0.62\right)$, whereas they were not significantly correlated in June or July.

(3) The variation trends of PAR and SST are approximately the same, and the PAR in this time period maintained a range of $40-50 \mathrm{~mol} /\left(\mathrm{m}^{2} \cdot \mathrm{d}\right)$. The energy provided by illumination was suitable for the growth and outbreak of Ulva prolifera. In addition, because of the abundant nutrients and suitable temperatures in the sea area near northern Jiangsu shoal, high growth rate of Ulva prolifera was observed in May.

In summary, the growth and outbreak of Ulva prolifera in the southern Yellow Sea were subject to the combined influence of multiple factors. In this study, using remote sensing satellite data, we analyzed the influence of SST on the outbreak of Ulva prolifera. In subsequent studies, we will combine more hydrological and environmental data (nutrients and sea surface wind) to conduct a multifactor comprehensive study of the life cycle of Ulva prolifera.

\section{References}

Cai Xiaoqing, Cui Tingwei, Zheng Ronger et al., 2014. Comparison of algorithms for green macro-algae bloom detection based on geostationary ocean color imager. Remote Sensing Information, 29(5): 44-50. (in Chinese)

Collini E, Wong C Y, Wilk K E et al., 2010. Coherently wired light-harvesting in photosynthetic marine algae at ambient temperature. Nature, 463(7281): 644-647. doi: 10.1038/nature 08811

Cui J J, Zhang J H, Huo Y Z et al., 2015. Adaptability of free-floating green tide algae in the Yellow Sea to variable temperature and light intensity. Marine Pollution Bulletin, 101(2): 660-666. doi: 10.1016/j.marpolbul.2015.10.033

Cui Linlin, Hu Song, Yang Hong et al., 2014. Weather process analysis for the period during the early aggregation of green tide. $M a-$ rine Environmental Science, 33(6): 941-946. (in Chinese)

Ding L P, Fei X G, Lu Q Q et al., 2009. The possibility analysis of habitats, origin and reappearance of bloom green alga (Enteromorpha prolifera) on inshore of western Yellow Sea. Chinese Journal of Oceanology and Limnology, 27(3): 421-424. doi: 10.1007/s00343-009-9277-x

Fan Shiliang, Fu Mingzhu, Li Yan et al., 2012. Origin and development of Huanghai (Yellow) sea green-tides in 2009 and 2010. Acta Oceanologica Sinica, 34(6): 187-194. (in Chinese)

Fan S L, Fu M Z, Wang Z L et al., 2015. Temporal variation of green macroalgal assemblage on Porphyra aquaculture rafts in the Subei Shoal, China. Estuarine, Coastal and Shelf Science, 163: 23-28. doi: 10.1016/j.ecss.2015.03.016

Fu Qianqian, Li Hangxiao, Wu Hailong et al., 2018. Effects of light intensity on photosynthetic physiology characteristic and short-time temperature reaction of Ulva linza. Oceanologia et Limnologia Sinica, 49(5): 967-974. (in Chinese)

Jin S, Liu Y X, Sun C et al., 2018. A study of the environmental factors influencing the growth phases of Ulva. prolifera in the southern Yellow Sea, China. Marine Pollution Bulletin, 135: 1016-1025. doi: 10.1016/j.marpolbul.2018.08.035

Keesing J K, Liu D Y, Fearns P et al., 2011. Inter- and intra-annual patterns of Ulva prolifera green tides in the Yellow Sea during 2007-2009, their origin and relationship to the expansion of coastal seaweed aquaculture in China. Marine Pollution Bulletin, 62(6): 1169-1182. doi: 10.1016/j.marpolbul. 2011.03.040

Kim J H, Kang E J, Park M G et al., 2011. Effects of temperature and irradiance on photosynthesis and growth of a green-tide-forming species (Ulva linza) in the Yellow Sea. Journal of Applied Phycology, 23(3): 421-432. doi: 10.1007/ s10811-010-9590-y

Lee J H, Pang I C, Moon I J et al., 2011. On physical factors that controlled the massive green tide occurrence along the southern coast of the Shandong Peninsula in 2008: a numerical study using a particle-tracking experiment. Journal of Geophysical Research, 116(C12): C12036. doi: 10.1029/ 2011JC007512

Li Deping, Yang Yuqiang, Dong Haiying et al., 2009. Cause analysis and synoptic characteristics for outbreaks of Enteromorpha around Qingdao in 2008. Periodical of Ocean University of China, 39(6): 1165-1170. (in Chinese)

Liu D Y, Keesing J K, Xing Q G et al., 2009. World's largest macroalgal bloom caused by expansion of seaweed aquaculture in China. Marine Pollution Bulletin, 58(6): 888-895. doi: 10.1016/j.marpolbul.2009.01.013

Liu M Y, Mao D H, Wang Z M et al., 2018. Rapid invasion of Spartina alterniflora in the coastal zone of mainland China: new observations from Landsat OLI images. Remote Sensing, 10: 1933. doi:10.3390/rs10121933

Liu X Q, Wang Z L, Zhang X L, 2016. A review of the green tides in the Yellow Sea, China. Marine Environmental Research, 119: 189-196. doi: 10.1016/j.marenvres.2016.06.004

Mao D H, Liu M Y, Wang Z M et al., 2019. Rapid invasion of Spartina alterniflora in the coastal zone of mainland: spatiotemporal patterns and human prevention. Sensors, 19: 2308. doi:10.3390/s19102308

Mao D H, Wang Z M, Du B J et al., 2020. National wetland mapping in China: a new product resulting from object-based and hierarchical classification of Landsat 8 OLI images. ISPRS Journal of Photogrammetry and Remote Sensing, 164: 11-25.

Mao D H, Wang Z M, Wu J G et al., 2018. China's wetlands loss to urban expansion. Land Degradation \& Development, 29: 2644-2657.

Park K A, Lee E Y, Chang E et al., 2015. Spatial and temporal 
variability of sea surface temperature and warming trends in the Yellow Sea. Journal of Marine Systems, 143: 24-38. doi: 10.1016/j.jmarsys.2014.10.013

Qi L, Hu C M, Xing Q G et al., 2016. Long-term trend of Ulva prolifera blooms in the western Yellow Sea. Harmful Algae, 58: 35-44. doi: 10.1016/j.hal.2016.07.004

Qiao Fangli, Wang Guansuo, Lü Xingang et al., 2011. Drift characteristics of green macroalgae in the Yellow Sea in 2008 and 2010. Chinese Science Bulletin, 56(21): 2236-2242. doi: 10.1007/s11434-011-4551-7

Shi X Y, Qi M Y, Tang H J et al., 2015. Spatial and temporal nutrient variations in the Yellow Sea and their effects on Ulva prolifera blooms. Estuarine, Coastal and Shelf Science, 163: 36-43. doi: 10.1016/j.ecss.2015.02.007

Sun Xiao, Wu Mengquan, He Fuhong et al., 2017. Temporal and spatial distribution of Ulva prolifera in the Yellow Sea and influence of typhoon 'CHAN-HOM' in 2015. Remote Sensing Technology and Application, 32(5): 921-930. (in Chinese)

Sun X, Wu M Q, Xing Q G et al., 2018. Spatio-temporal patterns of Ulva prolifera blooms and the corresponding influence on chlorophyll-a concentration in the southern Yellow Sea, China. Science of the Total Environment, 640-641: 807-820. doi: 10.1016/j.scitotenv.2018.05.378

Taylor R, Fletcher R L, Raven J A, 2001. Preliminary studies on the growth of selected 'green tide' algae in laboratory culture: effects of irradiance, temperature, salinity and nutrients on growth rate. Botanica Marina, 44(4): 327-336. doi: 10.1515/BOT.2001.042

Wang Kai, Ye Hong, Chen Feng et al., 2010. Long-term change of solar radiation in southeastern China: variation, factors, and climate forcing. Ecology and Environmental Sciences, 19(5): 1119-1124. (in Chinese)

Wang Ning, Huang Juan, Cui Tingwei et al., 2014. Capability comparison of 5 vegetation indices for detecting the green tide in different development phases and the application. Acta Laser Biology Sinica, 23(6): 590-595. (in Chinese)

Wang Zongling, Fu Mingzhu, Xiao Jie et al., 2018. Progress on the study of the Yellow Sea green tides caused by Ulva prolifera. Acta Oceanologica Sinica, 40(2): 1-13. (in Chinese)

Wei Q S, Yu Z G, Wang B D et al., 2016. Coupling of the spatial-temporal distributions of nutrients and physical conditions in the southern Yellow Sea. Journal of Marine Systems, 156: 30-45. doi: 10.1016/j.jmarsys.2015.12.001

Wei S, Li Y, Fang S et al., 2015. Temporal and spatial distributions of green algae micro-propagules in the coastal waters of the Subei Shoal, China. Estuarine, Coastal and Shelf Science, 163: 29-35. doi: 10.1016/j.ecss.2014.08.006

Wild M, Gilgen H, Roesch A et al., 2005. From dimming to brightening: decadal changes in solar radiation at earth's surface. Science, 308(5723): 847-850. doi: 10.1126/science. 1103215

Wu Mengquan, Guo Hao, Zhang Anding et al., 2014. Research on the characteristics of Ulva prolifera in Shandong Peninsula during 2008-2012 based on MODIS data. Spectroscopy and Spectral Analysis, 34(5): 1312-1318. (in Chinese)

Wu Qing, 2015. Mechanism of Floating and Sinking for Ulva Prolifera. Shanghai: Shanghai Ocean University. (in Chinese)

Xing Q G, Hu C M, Tang D L et al., 2015. World's largest macroalgal blooms altered phytoplankton biomass in summer in the Yellow Sea: satellite observations. Remote Sensing, 7(9): 12297-12313. doi: 10.3390/rs70912297

$\mathrm{Xu}$ Q, Zhang H Y, Ju L et al., 2014. Interannual variability of Ulva prolifera blooms in the Yellow Sea. International Journal of Remote Sensing, 35(11-12): 4099-4113. doi: 10.1080/01431161.2014.916052

Yi Li, Zhang Suping, Yin Yuqi, 2010. Influence of environmental hydro-meteorological conditions to Enteromorpha prolifera blooms in Yellow Sea, 2009. Periodical of Ocean University of China, 40(10): 15-23. (in Chinese)

Zhang Guangzong, Wu Mengquan, Sun Xiao et al., 2018. The inter-annual drift and driven force of Ulva prolifera bloom in the southern Yellow Sea. Oceanologia et Limnologia Sinica, 49(5): 1084-1093. (in Chinese)

Zhang J H, Kim J K, Yarish C et al., 2016. The expansion of Ulva prolifera O.F. Muller macroalgal blooms in the Yellow Sea, PR China, through asexual reproduction. Marine Pollution Bulletin, 104(1-2): 101-106. doi: 10.1016/j.marpolbul.2016. 01.056

Zhang Xiaohong, Wang Zongling, Li Ruixiang et al., 2012. Microscopic observation on population growth and reproduction of Entromorphra prolifera under different temperature and salinity. Advances in Marine Science, 30(2): 276-283. (in Chinese)

Zhao Chang, Yin Liping, Wang Guansuo et al., 2018. The modelling of Ulva prolifera transport in the Yellow Sea and its application. Oceanologia et Limnologia Sinica, 49(5): 1075-1083. (in Chinese)

Zhu Xudong, He Honglin, Liu Min et al., 2010. Spatio-temporal variation characteristics of photosynthetically active radiation in China in recent 50 years. Acta Geographica Sinica, 65(3): 270-280. (in Chinese) 